\title{
Interactions of von Willebrand Factor on Mica Studied by Atomic Force Microscopy
}

\author{
ROGER E. MARCHANT, ${ }^{*, 1}$ A. SCOTT LEA, $\dagger$ JOSEPH D. ANDRADE, $\dagger$ \\ AND PAULA BOCKENSTEDT, $;$ \\ * Department of Biomedical Engineering, Case Western Reserve University, Cleveland, Ohio 44106; $\uparrow$ Department of \\ Bioengineering, University of Utah, Salt Lake City, Utah 84112; \$Department of Internal Medicine, \\ University of Michigan, Ann Arbor, Michigan 48109
}

Received May 21, 1991 ; accepted July 3, 1991

\begin{abstract}
Three-dimensional molecular level images of uncoated human von Willebrand factor (vWF), a plasma glycoprotein, have been obtained using atomic force microscopy (AFM). However, the structural detail obtained by AFM was found to depend on the interactive forces between the hydrophilic mica surface and the vWF, relative to the tracking force of the probe tip acting on the protein. vWF on mica was characterized by AFM in air, under two deposition conditions, which differed with respect to the amount of vWF on the surface and the degree of sample hydration. Under hydrated conditions, the vWF had weak adhesion to the mica and was moved easily by the AFM probe tip. This resulted in tip-induced organization of $\mathrm{vWF}$ into molecular aggregates oriented approximately perpendicular to the fast scanning direction. In contrast, under dehydrated conditions, three-dimensional images of individual vWF molecules were observed at approximately $10 \mathrm{~nm}$ lateral resolution. Extended vWF molecules up to $600 \mathrm{~nm}$ in length were imaged, but most molecules were in the $50-300 \mathrm{~nm}$ range with $15-45 \mathrm{~nm}$ globular subunits. AFM images obtained using the constant force imaging mode indicated height measurements of 3-5 $\mathrm{nm}$ for most of the vWF molecules. @1992 Academic Press, Inc.
\end{abstract}

\section{INTRODUCTION}

The interactions of plasma proteins with solid surfaces are important phenomena that affect many bioadhesion events, including thrombus formation on biomedical polymers and other biomaterials (1-3). Thrombosis formation is a complex process that involves initial protein adsorption; the adhesion, activation, and aggregation of circulating platelets; and activation of coagulation pathways $(1,2)$. The composition, packing, orientation, and conformation of the adsorbed protein molecules are believed to be critical factors that determine subsequent events such as platelet and cell attachment (3-10). However, analysis or modeling of proteins at biomaterial surfaces is complicated significantly by a wide range of potential protein interactions and by the com-

${ }^{1}$ To whom correspondence should be addressed. plex and heterogeneous nature of most biomaterial surfaces (4-7). As a result, there is only limited fundamental understanding at the molecular level of the interactions of plasma proteins at solid surfaces.

Direct investigations of protein-surface interactions are now feasible by using the recently developed technique of atomic force microscopy (AFM) (11, 12). Indeed, under optimum conditions (e.g., hard, smooth samples) the AFM is capable of resolving surface detail down to the atomic level (11-14). Imaging can be accomplished in controlled atmosphere, air, or in aqueous environments, with no special requirement for high energy radiation sources or sample conductivity. AFM has been used to obtain atomic resolution of both conductors $(14,15)$ and hard nonconductors (16), and molecular level images of insulating polymers (17). AFM images of plasma proteins have been reported for fi- 
brinogen (18) imaged in air, and under buffer solutions for fibrinogen and thrombin activated polymerization of fibrin (19), monoclonal immunoglobulin (20), and supported lipid-protein membranes (21). In addition, AFM images of cells and surface features of cell membranes down to $20 \mathrm{~nm}$ resolution has been demonstrated (22).

The possibility of imaging the three-dimensional structure of biological molecules has stimulated considerable interest in the AFM technique and its potential use in the study of protein structure, protein adsorption, and protein-surface interactions. However, despite initial progress, AFM images of plasma proteins adsorbing to solid surfaces from aqueous buffer solutions generally have not shown molecular-level detail, but rather molecular aggregate formations in which very different proteins provide very similar "strand-like" images $(19,20)$. To address this issue, and with the aim of achieving molecular-level resolution, the AFM studies reported here focus on the effects of molecular probing in the repulsive mode on von Willebrand factor (vWF) deposited on mica and imaged under hydrated

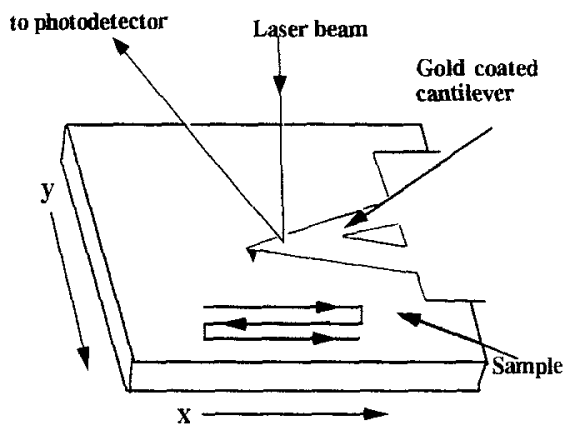

FIG. 1. Cantilever and AFM scanning. The sample surface is scanned with a sharp tip mounted on a cantilever. The small deflections of the cantilever are measured using a focused laser beam which is reflected off the gold-coated cantilever to a two-element photodiode detector. This allows for the precise measurement $(<0.1 \mathrm{~nm}$ ) in the $z$ direction. The $x, y, z$ piezoelectric single tube scanner, located directly under the sample, provides the precise raster movement of the sample. The variations in voltage signals from the photodetector and $z$-piezo are converted into a three-dimensional image. and dehydrated conditions. vWF is a very large plasma glycoprotein which exists in the circulation as polydisperse molecular assemblies of disulfide-linked multimers, ranging in molecular weight from 500,000 (dimer) up to 20 million (23). vWF serves as a carrier protein for coagulation factor VIII and as a mediator of initial platelet adhesion to substrata such as subendothelial collagen and possibly to implanted biomaterials. The three-dimensional structure of vWF is not known, but the glycoprotein has been visualized previously by TEM as a molecular filament (usually coiled) of varying sizes, typically 40 to $300 \mathrm{~nm}$ diameter when coiled, to over $1000 \mathrm{~nm}$ when fully extended (24-26). The large size of vWF and its crucial role as a mediator of thrombus formation on biomaterial surfaces made it an attractive protein for this initial AFM study.

\section{MATERIALS AND METHODS}

\section{Preparation of $v W F$}

Purified human vWF was prepared from cryoprecipitate and separated from coagulation factor VIII according to previously published methods (27). Briefly, this involved isolating the cryoprecipitate fractions with ristocetin-dependent platelet agglutinating activity. The purified $\mathrm{VWF}$ was suspended in TBS (50 $\mathrm{m} M$ Tris, $150 \mathrm{~m} M \mathrm{NaCl}, \mathrm{pH} \mathrm{7.4)}$, applied to a Sephacyl S-1000 column and eluted with the same buffer. Active vWF fractions were pooled, concentrated by precipitation with $35 \%(\mathrm{wt} / \mathrm{v})$ ammonium sulfate, centrifuged, and then suspended in TBS-azide buffer $(50 \mathrm{~m} M$ Tris, $150 \mathrm{~m} M \mathrm{NaCl}, 0.02 \%$ sodium azide, $\mathrm{pH}$ 7.4). Purified vWF fractions were diluted $(0.4$ and $0.04 \mathrm{mg} / \mathrm{mL})$ in TBSazide buffer and stored at $-20^{\circ} \mathrm{C}$.

\section{Atomic Force Microscopy}

AFM images of human vWF were obtained using a Digital Instruments AFM/STM Nanoscope II system. AFM operates by scanning a surface with a sharp tip mounted on a cantilever as shown in Fig. 1. For these ex- 
periments, a $12-\mu \mathrm{m} x-y$ scanner was used, which allows relatively large fields to be imaged. The AFM head includes a focused laser beam which is reflected off the upper apex surface of a gold-coated silicon nitride triangular cantilever to a two-element photodiode detector. The triangular cantilevers (Park Scientific Instruments) were $100 \mu \mathrm{m}$ in length with a spring constant of $0.37 \mathrm{~N} / \mathrm{m}$, and an integrated pyrimidal probe tip, approximately 2.7 $\mu \mathrm{m}$ in length and nominal tip radius of 30 $40 \mathrm{~nm}$. The optical beam detection allows for the precise measurement $(<0.1 \mathrm{~nm})$ in the $z$ direction of deflections by the cantilever and the attached probe tip. The sample stage mounting includes the $x, y, z$ piezoelectric single tube scanner, which provides the precise $x$ and $y$ raster movement of the sample. The image processing system converts the variations in voltage signals from the photodector and $z$-piezo, as a function of probe position, into a three-dimensional image.

\section{AFM Imaging of $v W F$}

AFM Images $(400 \times 400$ data pixel density $)$ were recorded in both the variable deflection (constant height) mode and in the constant force mode, where the applied force was kept constant by using an integral feedback loop and recording the $z$ movement of the sample. Atomic resolution images of freshly cleaved, muscovite mica, $\mathrm{KAl}_{3} \mathrm{Si}_{3} \mathrm{O}_{10}(\mathrm{OH})_{2}$, were obtained before imaging $v W F$. Samples were prepared by depositing a $2-\mu \mathrm{L}$ aliquot of $\mathrm{vWF}$ $(0.04 \mathrm{mg} / \mathrm{mL})$ onto the freshly cleaved mica. The aqueous drop spreads completely, since the mica surface is hydrophilic. Imaging of the vWF was initiated within a few minutes and referred to as hydrated conditions. For dehydrated conditions, vWF samples were removed from the piezoelectric stage, rinsed thoroughly with distilled water to remove loosely adherent molecules, air-dried for over $5 \mathrm{~h}$, and then reimaged. Thus, the two sample preparation conditions differed with respect to the amount of protein on the surface and the degree of hydration. AFM images were obtained over scan areas of 200 to $10,000 \mathrm{~nm}^{2}$, with typical $x$-scan frequencies of 3-9 Hz. Generally, the force applied (i.e., voltage setting, $V$ ) by the cantilever tip was minimized at the beginning of an experiment by using the deflection versus $z$ drive signal function. Under the minimum force conditions, the repulsive force plus the spring force approximately balances to within $5 \mathrm{nN}$ the attractive forces between probe and sample surface.

\section{RESULTS}

\section{vWF Imaged by AFM Under Hydrated Conditions}

The ability to obtain three-dimensional images of proteins on surfaces by AFM in the repulsive mode depends on the interactive forces between the two-dimensional solid surface and the protein, relative to the tracking force applied to the protein by the probe tip attached to the flexible cantilever. If the applied force, which can be minimized once scanning has been initiated, is greater than the surface-protein adhesive force, then the protein will be "moved" by the tip.

The effect of the probe tip is shown in Fig. 2 for sequential AFM images of vWF deposited on cleaved mica and then imaged using the constant height mode. Figure 2(a) shows an area (1368 $\mathrm{nm}$ by $1368 \mathrm{~nm}$ ) of deposited vWF that had not been scanned previously. The image shows molecular aggregates of vWF ranging from $15 \mathrm{~nm}$ up to $70 \mathrm{~nm}$ (width) dimension, which corresponds to $2-3 \mathrm{vWF}$ molecular diameters up to approximately $10 \mathrm{vWF}$ molecular diameters at the base of each aggregate strand. At low angles (approx. $30^{\circ}$ ) to the $x$ scanning direction, relatively thin aggregates of 15-20 nm were observed, which increased in size as the strand orientation approached $90^{\circ}$ to the $x$ axis. In the following scan (Fig. 2(b)), which was obtained under identical scanning conditions, the size range of the molecular aggregates increased to 30 $\mathrm{nm}$ at low angles to the $x$ direction, up to 90 $\mathrm{nm}$ in the perpendicular direction. In addition, the general orientation of the strands was 

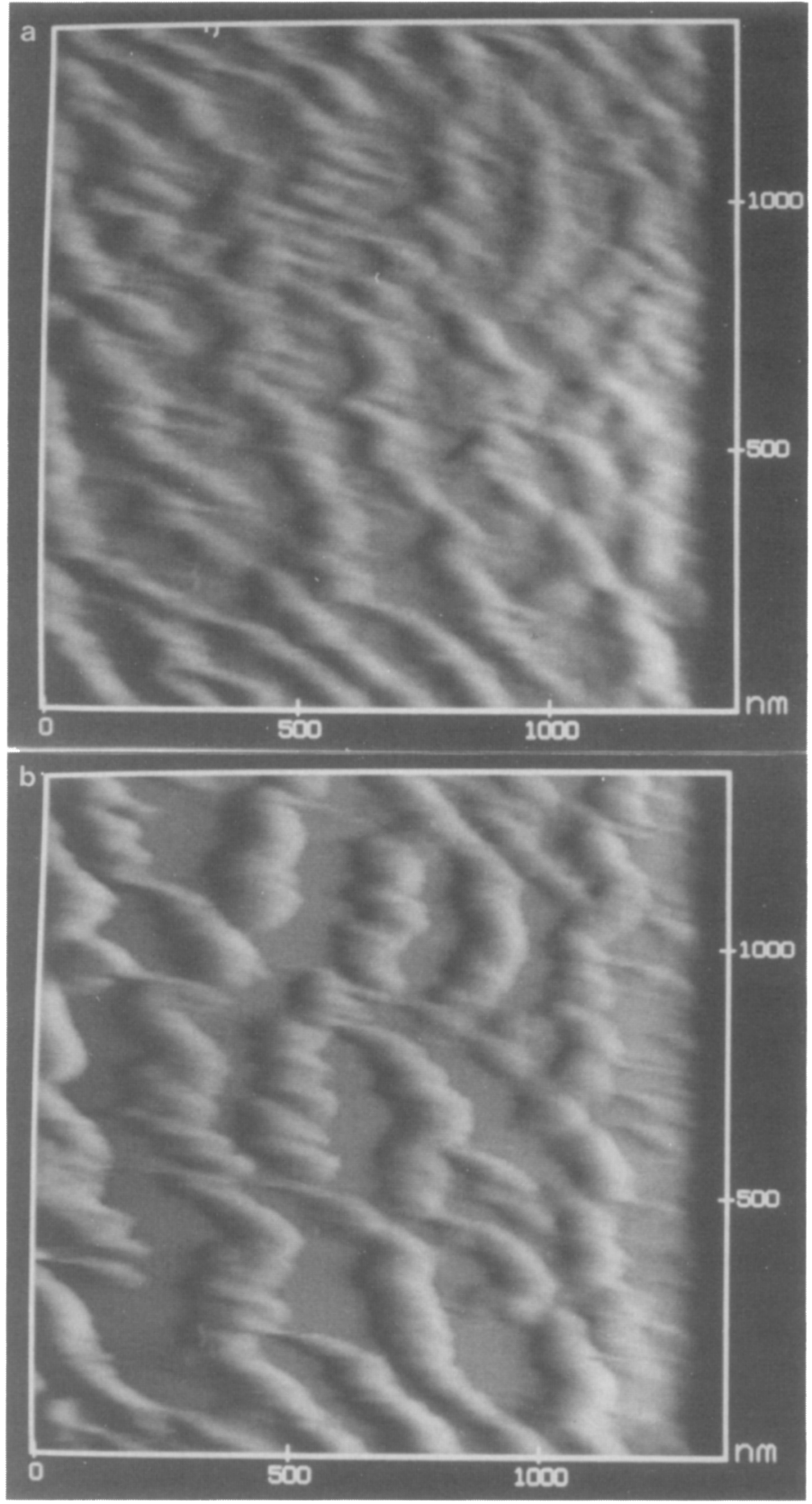

FIG. 2. Sequential constant height AFM images, $1368 \mathrm{~nm}$ by $1368 \mathrm{~nm}, 8.7 \mathrm{~Hz} x$-scan frequency, of vWF deposited on mica (hydrated conditions), which had not been scanned previously: (a) shows molecular aggregates of vWF ranging in size from $15 \mathrm{~nm}$ up to $70 \mathrm{~nm}$ (width dimension). In the following scan (b), the size range of the molecular aggregates has increased to $30 \mathrm{~nm}$ at low angles to the fast $(x)$ scanning direction up to $90 \mathrm{~nm}$ in the perpendicular direction. 
closer to the perpendicular direction, with the mica support evident between protein strands. Continued imaging caused further aggregation and manipulation of the vWF molecules. The height of the VWF aggregates was found to be approximately equal to the width of the growing molecular aggregates, determined from analysis of an image obtained using the feedback mode of constant force. The AFM images we obtained illustrated the effect of the probe tip, which was to move protein in the fast $(x)$ scanning direction until the resistance of the accumulated protein mass was sufficient to cause a deflection of the cantilever tip, thus providing an image of the aggregate strand oriented approximately $90^{\circ}$ to the $x$ axis.

The cumulative effect of protein manipulation by the probe tip was illustrated dra- matically, when the probed $\mathrm{vWF}$ was viewed at a higher scan area $(8 \times 8 \mu \mathrm{m})$, shown in Fig. 3, which provided a composite image of the earlier scanning effects. Area "a" in Fig. 3 has been scanned repeatedly, area " $b$ " was scanned 2-3 times, and area "c" shows undisturbed vWF. It should be noted that the fast scanning direction for area " $a$ " was the $y$ direction, as seen in Fig. 3. The whole area shown in Fig. 3 was scanned repeatedly, without perturbation to the vWF. In addition to illustrating the previous effects of the probe tip with scans, Fig. 3 shows that the tip interaction decreases with increasing full-scale scanning range, such that the vWF could be imaged without being disturbed by the probe tip, but at the cost of lower resolution. This may be attributed to an effect of the increased

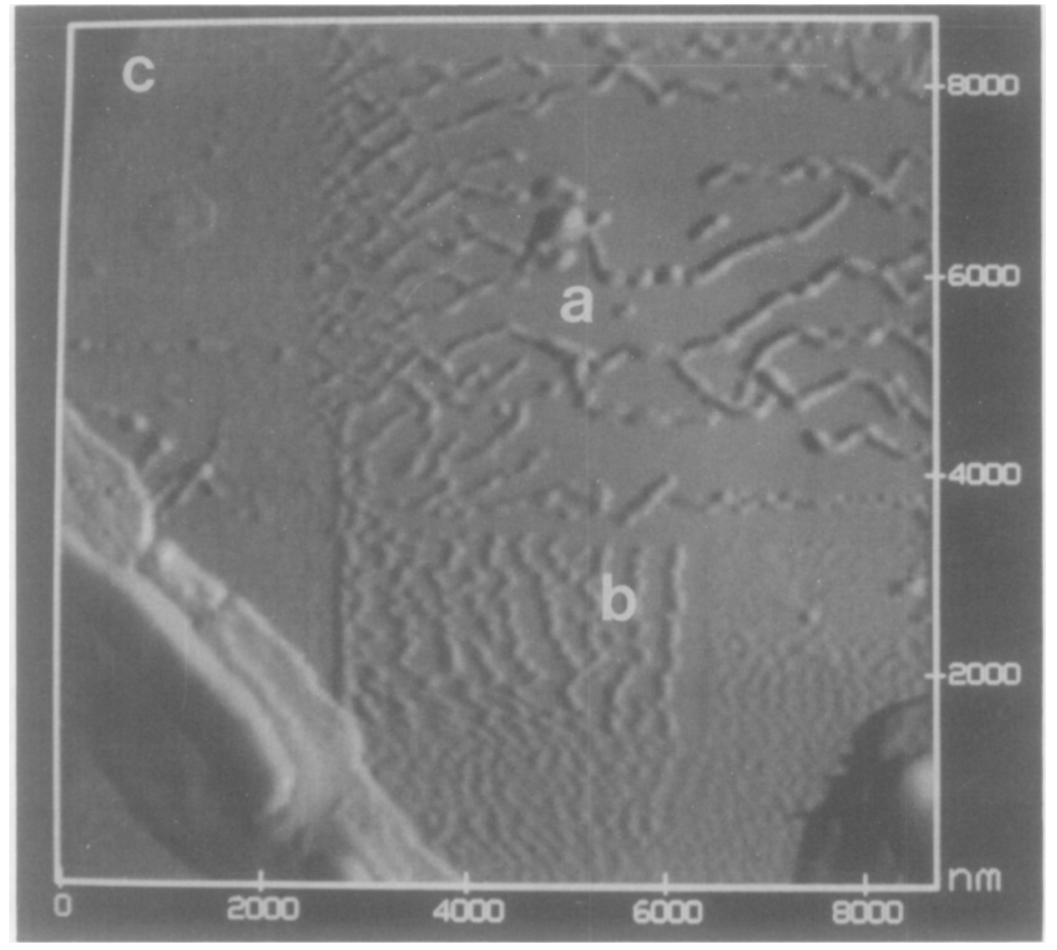

FIG. 3. AFM constant height image, $8664 \mathrm{~nm}$ by $8664 \mathrm{~nm}, 3.5 \mathrm{~Hz} x$-scan frequency, of vWF deposited on mica (hydrated conditions). The figure shows the cumulative effects of protein manipulation by the probe tip. Area " $a$ " has been scanned repeatedly ( 6 times), area " $b$ " has been scanned 2-3 times, and area " $c$ " shows undisturbed vWF. The $x$ axis was the fast scanning direction for areas " $b$ " and " $c$ " and the slow scanning direction for area "a." This large field image was scanned repeatedly without any effect on the protein. 
$x$ and $y$ scanning velocities $(\mathrm{nm} / \mathrm{s})$ at the higher scan size. Accordingly, our attempts to image vWF over a small full scale scan range such as $100 \mathrm{~nm}$, resulted in gross distortions to the protein layer. Although minimum force was used to image (and move) the vWF, we have no quantitative measure for the forces that were exerted locally by the tip on the vWF molecules, which is a function of tip geometry as well as the instrumental parameters described previously.

The cumulative effects of probing the hydrated vWF with the number of scans are summarized in Table I. The table includes information derived from additional scans that are not presented in this report. The table shows that the surface coverage of vWF on mica was reduced with each scan, from the initial $100 \%$ down to $39 \%$ after 6 scans, while the molecular aggregates increased in size from $15 \mathrm{~nm}$ up to $120 \mathrm{~nm}$.

\section{vWF Imaged by AFM Under Dehydrated Conditions}

To increase the vWF-mica adhesive interactions, samples were rinsed with distilled water to remove loosely adherent molecules, de-

\section{TABLE I}

The Effect of AFM Scans on Deposited vWF

\begin{tabular}{lcccc}
\hline $\begin{array}{c}\text { Number } \\
\text { of scans }^{a}\end{array}$ & $\begin{array}{c}\text { Scan } \\
\text { range } \\
(\mathbf{n m})\end{array}$ & $\begin{array}{c}\text { Scan } \\
\text { orientation }^{\left(\text {degres }^{b}\right.}\end{array}$ & $\begin{array}{c}\text { vWF Aggregates } \\
\text { size range } \\
(\mathbf{n m})\end{array}$ & $\begin{array}{c}\text { Surface } \\
\text { coverage } \\
\text { of vWF } \\
\text { on mica }^{d}\end{array}$ \\
\hline $1(2 \mathrm{a})$ & 1368 & 0 & $15-65$ & $>74 \%$ \\
$2(2 \mathrm{~b})$ & 1368 & 0 & $30-90$ & 61 \\
2 & 3723 & 0 & $30-110$ & 65 \\
3 & 3723 & 0 & $90-110$ & 52 \\
5 & 5165 & 90 & $110-130$ & 41 \\
6 & 5165 & 90 & $110-140(120)$ & 39 \\
\hline $6(3)$ & 8664 & 0 & & -
\end{tabular}

\footnotetext{
${ }^{a}$ Figure number in parentheses.

${ }^{b}$ Scan orientation is with respect to Fig. 3 .

${ }^{c}$ The maximum height (nm) of the vWF aggregates is shown in parentheses for the constant force image. The width of the vWF aggregates was obtained using the Nanoscope image review software.

${ }^{d}$ Surface coverage of vWF on mica was estimated from weighing cutouts of figure enlargements.
}

hydrated by air-drying for over $5 \mathrm{~h}$, and then re-imaged. Under these sample preparation conditions, individual vWF molecules were observed that were not perturbed by repeated scanning of the cantilever tip. In addition, this permitted additional time to sequentially scan identical areas using the constant height mode and then the constant force imaging mode.

AFM images of the dehydrated vWF molecules were obtained from full-scale scan areas ranging from of $200 \mathrm{~nm}^{2}$ up to $10,000 \mathrm{~nm}^{2}$. Figures 4(a) and 4(b) show sequential AFM images, $2600 \mathrm{~nm}$ by $2600 \mathrm{~nm}$, of $\mathrm{vWF}$ on mica, and imaged under constant height and then constant force. Analysis of these and similar large field images showed vWF molecules up to $600 \mathrm{~nm}$ in length, but molecular sizes in the range 50 to $300 \mathrm{~nm}$ were most common. These images confirm the considerable variation in size and shape of the vWF macromolecules, which result from the polydispersity and chain folding. Analysis of AFM images obtained using constant force such as Fig. 4(b), indicated apparent height measurements of 3-11 nm (1-2 vWF molecules), but with 3-5 nm ( $1 \mathrm{vWF}$ molecule) being the most common.

Figures 5(a) and 5(b) show two higher magnification constant height images (1161 $\mathrm{nm}$ by $1161 \mathrm{~nm}$ ) that show the typical range of observed vWF molecules. Figure 5(a) shows two relatively extended chains approximately $500 \mathrm{~nm}$ end-to-end length, while the largest molecule shown in Fig. 5(b) has a maximal dimension of $200 \mathrm{~nm}$. The two vWF molecular structures shown in Fig. 5(a) are not fully extended, but include regions with overlapping chains. This interpretation is consistent with the observed variations in molecular height measurements obtained from the companion constant force image. In addition, several regions of the two chains, examples are indicated by arrows, are suggestive of the vWF protomer (dimeric repeating unit) observed previously by $\operatorname{TEM}(24,25)$. One such area of Fig. 5(a) has been expanded and is shown in Fig. 6. The observed "globular" subunits seen in Figs. 5 and 6 range in size from $15-45 \mathrm{~nm}$. 


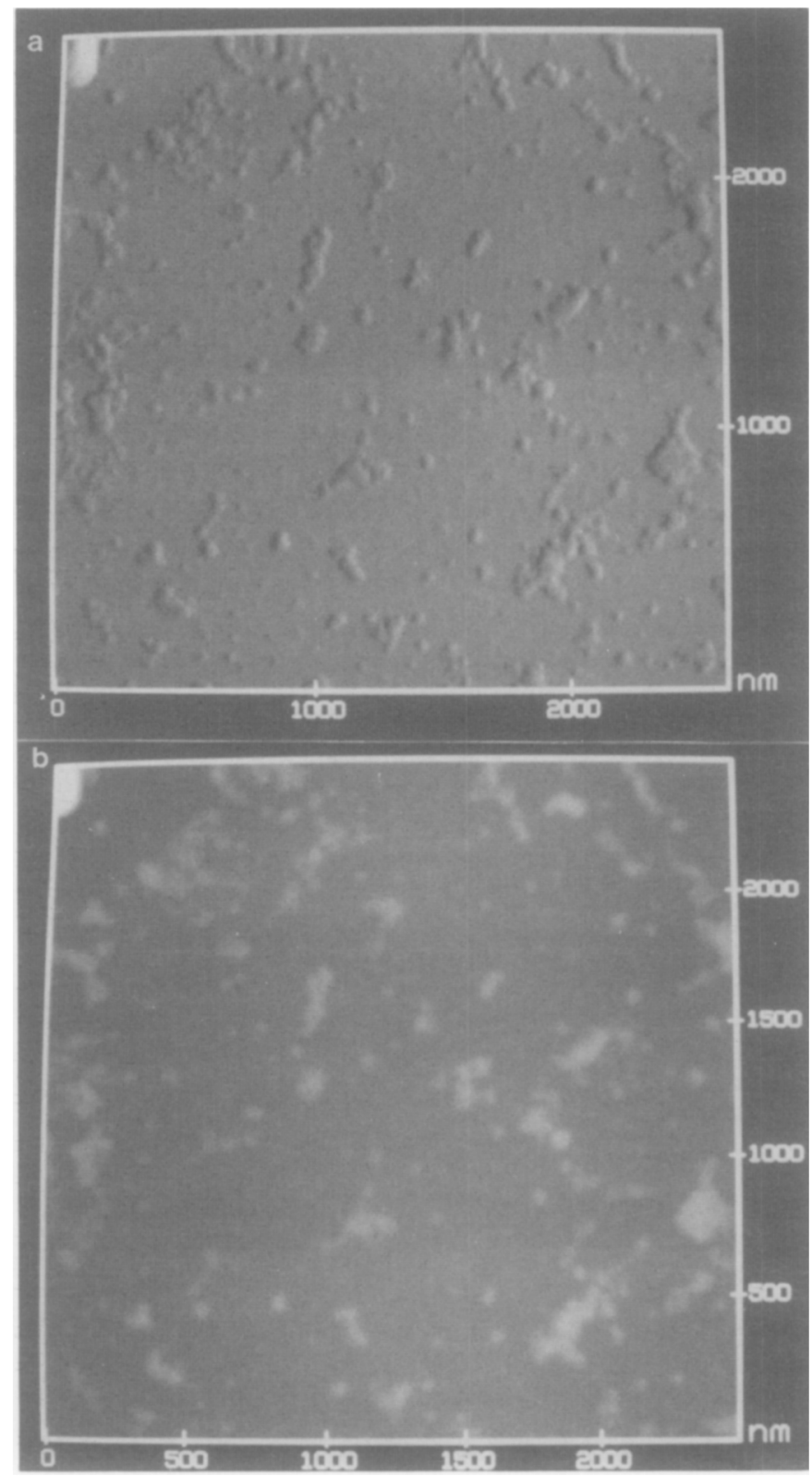

FIG. 4. Sequential constant height (a) and constant force (b) AFM images, $2600 \mathrm{~nm}$ by $2600 \mathrm{~nm}, 5.8$ $\mathrm{Hz} x$-scan frequency, of vWF deposited on mica; water rinsed and dried in air for over $5 \mathrm{~h}$ (dehydrated conditions). The images show vWF molecules with a wide range in lateral dimensions, from $50 \mathrm{~nm}$ to over $600 \mathrm{~nm}$. Height measurement of the molecules, obtained from (b), indicated a range of $3-11 \mathrm{~nm}$.

\section{DISCUSSION}

AFM Scanning Effects on $v W F$

The AFM images we obtained under hydrated conditions showed that the VWF mol- ecules were moved easily by the probe tip into a perpendicular orientation to the fast scanning direction, and subsequently to the peripheral edges of the scanning area, although we noted that the effects of the probe tip were 


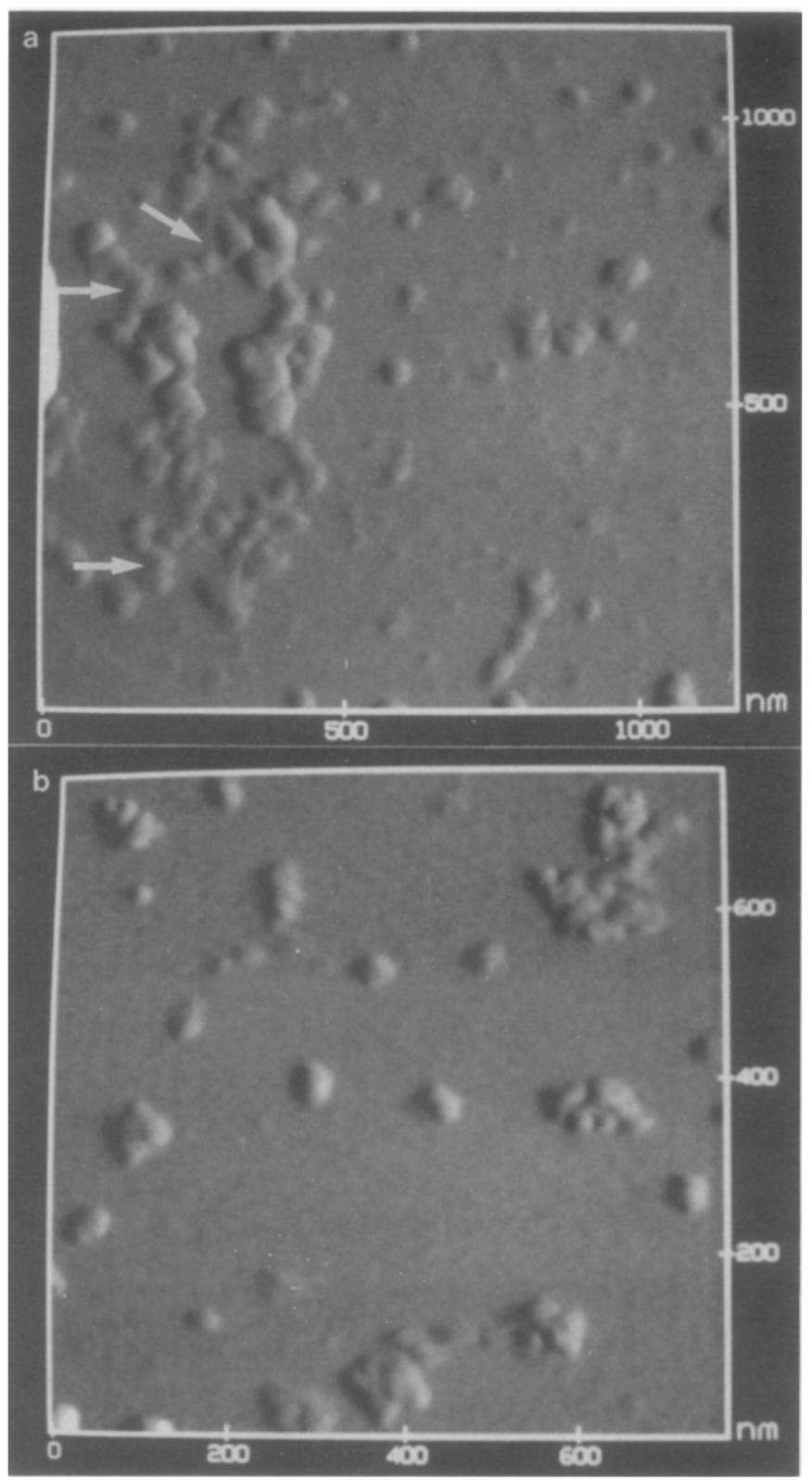

FIG. 5. Two AFM constant height images of VWF deposited on mica (dehydrated conditions): (a) (1161 $\mathrm{nm}$ by $1161 \mathrm{~nm}, 5.7 \mathrm{~Hz} x$-scan frequency) shows two partially extended vWF macromolecular chains, approx $500 \mathrm{~nm}$ end-to-end length. Several substructures of these chains, examples are indicated by arrows, are suggestive of the vWF protomer. The largest vWF molecule shown in (b) has a maximal dimension of approximately $200 \mathrm{~nm}$.

reduced significantly when the surface was scanned at higher scan width and velocity. The extent of the effects of the probe tip will depend on the properties of the support surface, the properties of the protein, the deposition conditions, and on the AFM scanning conditions. Each of these factors may be adjusted for the purposes of a particular experiment. Under 


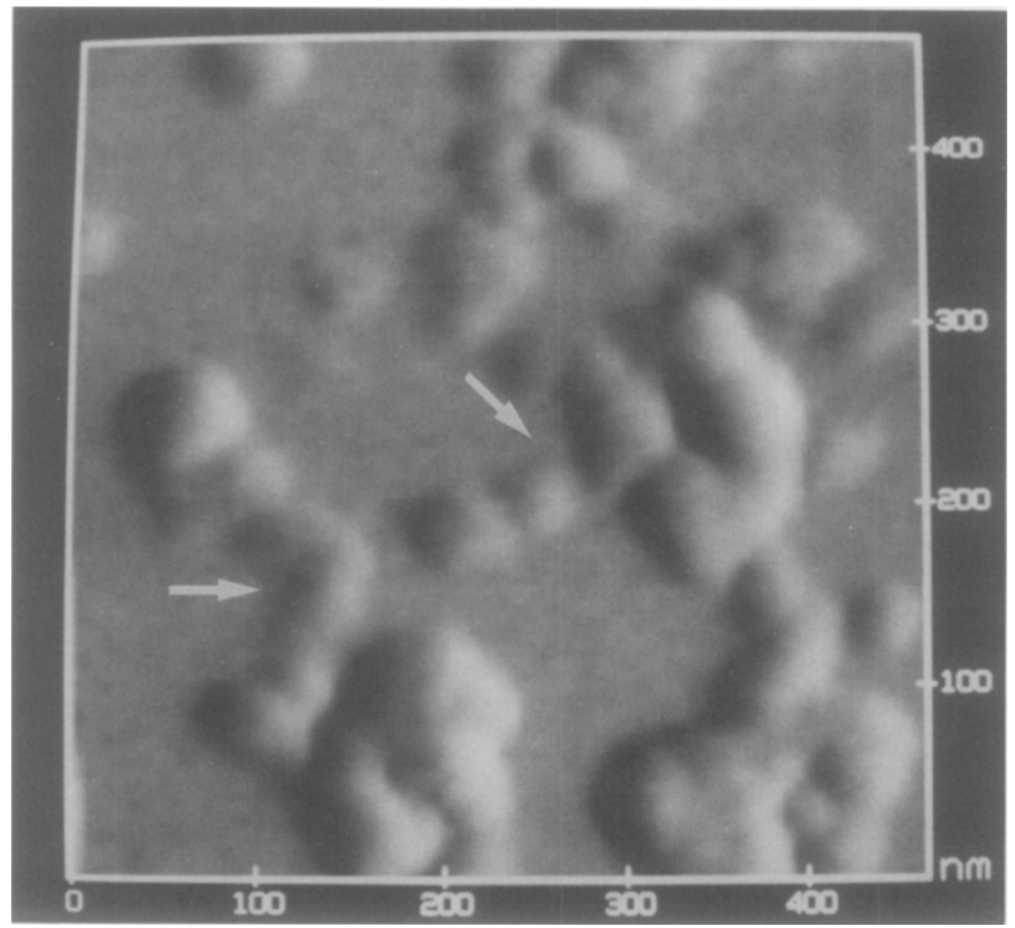

FIG. 6. Expanded AFM constant height image, $462 \mathrm{~nm}$ by $462 \mathrm{~nm}, 5.8 \mathrm{~Hz} x$-scan frequency of vWF deposited on mica (dehydrated conditions). The image shows two substructures (arrows) of larger vWF chains thought to be the vWF protomer region (dimeric repeating unit).

the initial conditions employed here, the vWF molecules had relatively weak and/or few adhesive contacts to the hydrophilic mica. The weak adhesion is attributed to the presence of water molecules at the vWF-mica interface, as depicted in the model of scanning effects on vWF, shown in Fig. 7(a).
The result of the probe tip moving small amounts of vWF into larger molecular aggregates are the sequential images shown in Fig. 2 . These images are very similar to other proteins observed under aqueous conditions ( 19 , 20) using an AFM liquid cell attachment. Although no structural information can be ob-

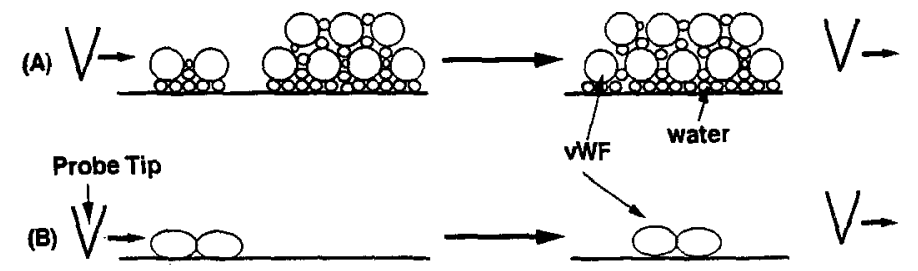

FIG. 7. Proposed models for AFM probing of vWF, under (A) hydrated conditions and (B) dehydrated conditions. Under hydrated conditions, the vWF-mica interactions are weak, attributed to the presence of interfacial water molecules. The weak interactions allows the probe tip to move protein molecules in the fast $(x)$ scanning direction into large molecular aggregates. The mass of the accumulated protein is then sufficient to cause a deflection of the cantilever (variable deflection mode), leading to molecular aggregates that are oriented perpendicular to the fast scanning direction. In the absence of interfacial water (B) the vWF molecules have stronger and increased surface contacts, which allowed repeated scanning by the AFM probe without significant perturbation to the vWF. 
tained from the images, a tentative analogy may be drawn from our present observations to protein adsorption behavior in an aqueous environment. The adsorption free energy of a protein attracted to an interface generally exceeds thermal energies leading to a quasi-irreversible attachment, although protein exchange reactions have been observed experimentally. Our data suggest that it is the loss of water molecules at the interface between the protein and solid surface which is the critical first step in the time dependent adsorption process. Thus, in the case of a hydrophobic surface, where attractive protein-surface interactions result in entropically driven exclusion of interfacial water and strong surfaceprotein adhesion, molecular resolution of the adsorbed protein by AFM should be possible. Conversely, an adsorbing protein that remains solvated will more readily desorb or undergo exchange reactions, or in the case of our experiments, be moved by the AFM probe tip. This view is supported by the ease with which most of the vWF molecules were rinsed off the mica surface.

Under dehydrated conditions, the vWF had stronger and/or increased surface contacts, caused by elimination of the interfacial and intermolecular water molecules, as shown in the model (Fig. 7(b)). Clearly, the loss of water at the interface had an important effect on the mica-vWF adhesion, which resulted in a significant improvement in our ability to successfully image the vWF molecules, down to approximately $10 \mathrm{~nm}$ lateral resolution and 1 $\mathrm{nm}$ vertical resolution. The images obtained by AFM are a convolution of tip and sample, where the ultimate resolution depends on the radius of curvature of the microfabricated tip, nominally estimated to be $30-40 \mathrm{~nm}$, but may be as small as $0.3 \mathrm{~nm}$ for an ultrasharp tip (28). The elliptical shape of the schematic vWF molecules is intended to reflect the uncertainty associated with our tip geometry, and for the possibility of some molecular spreading resulting from surface tension effects during water evaporation. The application of sample drying techniques used successfully in electron microscopy, can be expected to remove uncertainty associated with sample deformability and surface tension effects, leading ultimately to atomic scale images of plasma protein molecules. An alternative approach, which can be used for imaging structural features of biological macromolecules under aqueous conditions, is to immobilize the biomolecule (to mica or glass) via an appropriate coupling molecule (e:g., (21)). However, this approach then precludes the study of specific proteinsurface interactions, and the examination of the relationships between material surface properties and three-dimensional protein structure.

\section{Schematic Models of The vWF Protomer}

The vWF molecules observed by AFM under dehydrated conditions covered the range of maximal dimensions that were consistent with the published TEM data for coiled vWF $(24,25)$. TEM and quasi-elastic light scattering studies (25) of purified human vWF have shown that the maximal dimensions of purified human vWF in compact coils, which contribute greater than $87 \%$ of the molecules, range from $40 \mathrm{~nm}$ up to $300 \mathrm{~nm}$, while extended vWF molecules range from $100 \mathrm{~nm}$ up to $1100 \mathrm{~nm}$. In addition, vWF deposited on mica retains a similar statistical distribution of overall dimensions compared with vWF observed in buffer solution (26).

The three-dimensional structure of vWF is not known. However, by TEM data (24), the dimeric repeating unit (protomer) has been suggested to consist of two large globular end domains with an average size of $26 \mathrm{~nm}$, each consisting of two smaller $13 \mathrm{~nm}$ globular domains, which are connected to a small central node by two flexible rod domains each $34 \mathrm{~nm}$ by $2 \mathrm{~nm}$. The overall length of a fully extended vWF protomer is $100-120 \mathrm{~nm}$, as depicted in the schematic model shown Fig. 8(a). Figure 8 (b) shows similar schematic representations for two vWF structures observed by AFM (from Fig. 6), in which the solid spheres represent the globular domains. Based on con- 
(B)

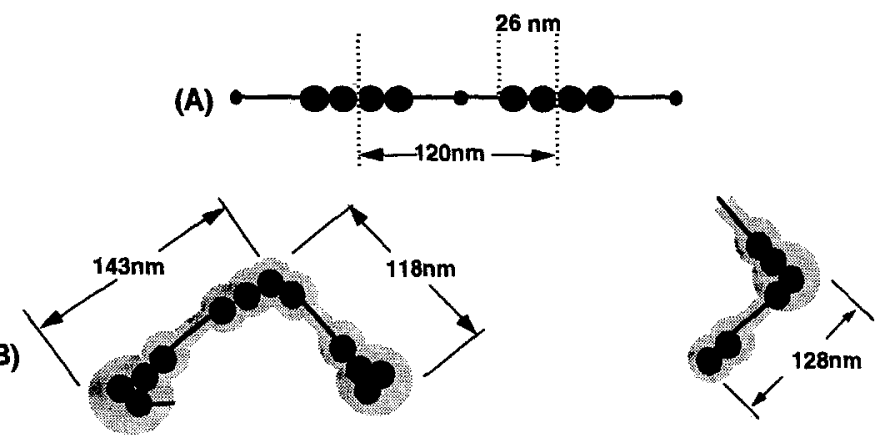

FIG. 8. Schematic models of the vWF protomer (dimer) region, (A) based on TEM data (after Ref. (23) and (B) based on AFM data (from Fig. 6). The solid spheres represent globular domains.

stant force images, the height of the largest globular domain, shown on the left of Fig. 8(b) is $7.5 \mathrm{~nm}$, while the height of the remaining globular domains in each structure is approximately $4 \mathrm{~nm}$. The $2-\mathrm{nm}$ internodal strands were not resolved by AFM in this study. The observed globular subunits range in diameter from $15-45 \mathrm{~nm}$. The larger sized subunits suggest some degree of overlap between globular domains at the interprotomer junction, as suggested by the TEM studies of Fowler $e t$ al. (24), but may also reflect the presence of the carbohydrate side chains which are believed to be clustered on the globular domains (23).

\section{SUMMARY}

These studies have shown that three dimensional molecular level images of uncoated human vWF glycoprotein molecules can be achieved using AFM. However, the structural detail obtained by AFM was shown to depend on the interactive forces between the two-dimensional mica surface and the $\mathrm{vWF}$, relative to the tracking force applied to the protein by the AFM probe tip. Initial experiments of hydrated vWF on mica showed that the applied force exerted by the probe tip was sufficient to manipulate the proteins into large molecular aggregates oriented perpendicular to the fast scanning direction. These observations were attributed to the weak $\mathrm{VWF}$-mica interactions resulting from the presence of interfacial water molecules. Air drying the vWF for an extended period was sufficient to permit molecular level imaging. Under these dehydrated conditions, images of $\mathrm{VWF}$ at approximately $10 \mathrm{~nm}$ lateral resolution and $1 \mathrm{~nm}$ vertical resolution were obtained. Extended vWF molecules up to 600 $\mathrm{nm}$ in length were imaged, but most molecules were in the 50-300 nm range with 15-45 nm globular subunits. AFM images obtained using the constant force imaging mode indicated height measurements of 3-5 $\mathrm{nm}$ for the globular subunits. The presence of larger sized subunits provided evidence for overlap between globular domains at the interprotomer (dimer) junction. Two substructures of a partially extended $v W F$ macromolecule observed by AFM were modeled and compared with an existing model for the dimeric repeating unit of $\mathrm{vWF}$.

\section{ACKNOWLEDGMENTS}

This work was supported in part by the National Institutes of Health, Grant HL-40047 (REM), Clinical Investigator Award IK08HL01789-01 (PB), and the Center for Bioploymers at Interfaces at the University of Utah. The authors thank Dr. A. Pungor for his comments and technical assistance with the AFM experiments and Drs. D. Ginsburg, C. C. Williams, and S. Eppell for their valuable comments during the preparation of this manuscript.

\section{REFERENCES}

1. Salzman, E. W., and Merrill, E. W., in "Hemostasis and Thrombosis" (R. W. Colman, J. Hirsh, V. J. Marder, and E. W. Salzman, Eds.), 2nd ed., p. 1335. Lippincott Co., Philadelphia, 1987.

Joumal of Colloid and Interface Science, Vol. 148, No. 1, January 1992 
2. Anderson, J. M., and Marchant, K. K., CRC Crit. Rev. Biocompat. 1, 111 (1985).

3. Chuang, H. Y. K., in "Blood Compatibility" (D. F. Williams, Ed.), Vol. 1, p. 87. CRC Press, Boca Raton, FL, 1987.

4. Andrade, J. D., in "Surface and Interfacial Aspects of Biomedical Polymers: Protein Adsorption" (J. D. Andrade, Ed.), Vol. 2, p. 1. Plenum Press, New York, 1985.

5. Giroux, T. A., and Cooper, S. L., J. Colloid Interface Sci. 139, 351 (1990).

6. Goodman, S. L., Simmons, S. R., Cooper, S. L., and Albrecht, R. M., J. Colloid Interface Sci. 139, 561 (1990).

7. Lundstrom, I., and Elwing, H., J. Colloid Interface Sci. 136, 68 (1990).

8. Slack, S., and Horbett, T. A., J. Colloid Interface Sci. 133, 148 (1989).

9. Nygren, H., and Stenberg, M., J. Biomed. Mater. Res. 22, 1 (1988).

10. Lewandowska, K., Kaetzel, C. S., Zardi, L., and Culp, L. A., FEBS Lett. 237, 35 (1988).

11. Binnig, G., Quate, C. F., and Gerber, C., Phys. Rev. Lett. 56, 930 (1986).

12. Hansma, P. K., Elings, V. B., Marti, O., and Bracker, C. E., Science (Washington, D.C. 1883-) 242, 209 (1988).

13. Schardt, B. C., Yau, S.-L., and Rinaldi, F., Science (Washington, D.C., 1883-) 243, 1050 (1989).

14. Manne, S., Hansma, P. K., Massie, J., Elings, V., and Gewirth, A. A., Science (Washington, D.C., 1883) 251, 183 (1991).
15. Marti, O., Drake, B., and Hansma, P. K., Appl. Phys. Lett. 51, 484 ( 1987).

16. Albrecht, T. R., and Quate, C. F., J. Appl. Phys. 62, 2599 ( 1987).

17. Marti, O., Ribi, H. O., Drake, B., Albrecht, T. R., Quate, C. F., and Hansma, P. K., Science (Washington, D.C., 1883-) 239, 50 (1988).

18. Wigren, A., et al., FEBS Lett. 280, 225 (1991).

19. Drake, B., Prater, C. B., Weisenhorn, A. L., Gould, S. A. C., Albrecht, T. R., Quate, C. F., Cannell, D. S., Hansma, H. G., and Hansma, P. K., Science (Washington, D.C., 1883-) 243, 1586 (1989).

20. Lin, J. N., Drake, B., Lea, A. S., Hansma, P. K., and Andrade, J. D., Langmuir 6, 509 (1990).

21. Weisenhorn, A. L., Drake, B., Prater, C. B., Gould, S. A. C., Hansma, P. K., Egger, M., Heyn, S. P., and Gaub, H. E., Biophys. J. 58, 1251 (1990).

22. Gould, S. A. C., Drake, B., Prater, C. B., Weisenhorn, A. L., Hansma, H. G., and Hansma, P. K., J. Vac. Sci. Technol. 8, 369 (1990).

23. Girma, J-P., Meyer, D., Verweij, C. L., Pannekoek, H., and Sixma, J. J., Blood 70, 605 (1987).

24. Fowler, W. E., Fretto, L. J., Hamilton, K. K., Erickson, H. P., and McKee, P. A., J. Clin. Invest. 76, 1491 (1985).

25. Slayter, H., Loscalzo, J., Brockenstedt, P., and Handin, R. I., J. Biol. Chem. 260, 8559 (1985).

26. Ohmori, K., Fretto, L. J., Harrison, R. L., Switzer, M. P., Erickson, H. P., and McKee, P. A., J. Cell Biol. 95, 632 (1982).

27. Bockenstedt, P., Greenberg, J., and Handin, R. I., J. Clin. Invest. 77, 743 (1986).

28. Williams, C. C., Personal Communication, University of Utah, 1991. 